\title{
Effects of response time deadlines on adults' strategy choices for simple addition
}

\author{
JAMIE I. D. CAMPBELL and SHAUNA AUSTIN \\ University of Saskatchewan, Saskatoon, Saskatchewan, Canada
}

\begin{abstract}
In this investigation of adults' solution strategies for simple arithmetic, participants solved addition problems (e.g., $2+3,8+7$ ) under fast and slow response deadlines: The participants were instructed either to respond before a 750-msec warning beep, or to wait for a 2,500-msec beep before responding. After each trial, they indicated whether they had solved the problem by direct memory retrieval or by using a procedural strategy (e.g., counting, transformation). It was predicted that the fast deadline condition should curtail the use of procedural strategies, which generally are slower than direct retrieval. Furthermore, this deadline effect should be exaggerated for numerically larger problems because procedural strategiesare especially slow for the largerproblems. As predicted, we observed a deadline $\times$ size interaction whereby the fast deadline increased reported use of retrieval, especially for large problems. The results confirm that reported use of direct retrieval decreases systematicallywith elapsed time, and they provide additional evidence that young, educated adults rely substantially on procedural strategies even for simple addition.
\end{abstract}

Understanding the cognitive processes of simple arithmetic (e.g., $3+4=$ ?, $9 \times 6=$ ?) is important, both because of its status as a fundamental intellectual skill and because arithmetic tasks are used widely in behavioral research and assessment. Until recently, it was commonly assumed that educated adults in North America relied more or less exclusively on direct memory retrieval to solve single-digit addition and multiplication problems such as $5+6$ or $4 \times 8$. Indeed, much of the cognitive research on adults' basic arithmetic over the last 30 years has focused almost exclusively on modeling the associative memory processes involved (Ashcraft, 1992, 1995). In many recent studies, however, researchers have concluded that educated adults do not rely exclusively on memory for simple arithmetic and instead may often use procedural methods based on counting or transformation (e.g., Campbell \& Xue, 2001; Geary, Frensch, \& Wiley, 1993; LeFevre, Bisanz, et al., 1996; LeFevre \& Liu, 1997; LeFevre, Sadesky, \& Bisanz, 1996).

This revision in the theoretical conception of adults' basic arithmetic skills is based largely on strategy self-reports, but relatively little effort has been expended to evaluate their validity. The general strategy for validation has been to show that performance varies appropriately across different reported strategies. For example, for simple addition, LeFevre, Sadesky, and Bisanz (1996) found that direct memory retrieval was the dominant strategy reported by Canadian university students for small-number problems (e.g., about $85 \%$ of trials for problems with a sum $\leq 10$ ).

This research was supported by a grant from the Natural Sciences and Engineering Research Council of Canada. Address correspondence to J. I. D. Campbell, Department of Psychology, University of Saskatchewan, 9 Campus Drive, Saskatoon, SK, S7N 5A5 Canada (e-mail: jamie.campbell@ usask.ca).
For larger problems, however, the students reported a variety of procedural solution strategies, which they used about half the time to solve the large problems. One would expect greater use of direct retrieval for small problems than for larger problems, because small-number problems are encountered more frequently and therefore are more likely to have high memory strength relative to large problems (Ashcraft \& Christy, 1995; Geary, 1996; Hamann \& Ashcraft, 1986).

Other evidence validating the strategy reports emerged through LeFevre, Sadesky, and Bisanz's (1996) analysis of the problem size effect, the common finding that the difficulty of simple arithmetic problems increases with numerical size. LeFevre, Sadesky, and Bisanz pointed out that the problem size effect for addition retrieval trials was much smaller than on trials where participants had reported using a procedure. This would be expected because, whereas procedural strategies generally ought to be slower than retrieval, this difference would be exaggerated for large problems: Applying procedural strategies for largenumber problems typically involves more steps or more difficult steps than does applying procedural strategies for small-number problems. The overall strategy use and performance pattern observed by LeFevre, Sadesky, and Bisanz (i.e., direct retrieval is more likely for small than for large problems; retrieval trials are fast relative to procedure trials; the problem size effect on RT is smaller for retrieval than for procedure trials) has since been observed in numerous experimental studies involving all four basic arithmetic operations (e.g., Campbell \& Fugelsang, 2001; Campbell \& Timm, 2000; Campbell \& Xue, 2001; Hecht, 1999; LeFevre \& Morris, 1999; Robinson, 2001).

Although the performance patterns associated with arithmetic strategy reports lend them credibility, there are good reasons to be concerned about their validity. Kirk 
and Ashcraft (2001) demonstrated that adults' strategy reports for simple addition and multiplication are very sensitive to instructional biases. Specifically, instructions that emphasized normative use of retrieval resulted in dramatically increased reports of retrieval relative to a no-bias condition, whereas instructions that emphasized normative use of procedural strategies resulted in decreased reports of retrieval. Addition response time (RT) performance of the retrieval-biased group most closely resembled a silent (i.e., no report) control group, suggesting that even the supposedly unbiased instructions about use of multiple strategies reduced reports of direct retrieval. Such findings motivate further experimental investigation into adults' strategies for simple arithmetic and the validity of strategy self-reports.

\section{The Present Study}

Here, we attempted to directly manipulate adults' strategies for simple addition (e.g., $2+3,4+8$ ) by imposing a time limit $(750 \mathrm{msec})$ that made it difficult to use a procedural strategy (e.g., counting or transformation), contrasting this with a condition where participants had to wait 2,500 msec before responding. The results of LeFevre, Sadesky, and Bisanz (1996, p. 222) suggest that $750 \mathrm{msec}$ is faster than most people can complete a procedural strategy for addition, whereas addition retrieval can often be achieved by this deadline. In contrast, $2,500 \mathrm{msec}$ should be adequate for one to solve most simple addition problems using either retrieval or procedural strategies. After each arithmetic trial, our participants identified their strategies by selecting from a list that included Transform, Count, Remember, and Other as options (cf. Campbell \& Timm, 2000; Campbell \& Xue, 2001). These options correspond to categories of simple arithmetic strategies identified in previous research using a full-report method in which participants described their specific activities after each trial (LeFevre, Bisanz, et al., 1996; LeFevre, Sadesky, \& Bisanz, 1996).

If participants are generally able to discriminate use of direct retrieval (i.e., Remember) from use of procedural strategies (i.e., Count, Transform), we would expect the following results. The 750-msec deadline condition should curtail use of procedural strategies, because it would be too fast to permit completion of most calculation procedures. The fast deadline therefore should force participants to attempt to use retrieval. Furthermore, this deadline effect should be exaggerated for numerically larger problems, because procedural strategies are especially slow for the larger problems (e.g., Campbell \& Xue, 2001; LeFevre, Sadesky, \& Bisanz, 1996). Thus, we predicted a deadline $X$ size interaction wherein the fast deadline would produce an increase in retrieval reports relative to the slow deadline, especially for larger problems.

\section{METHOD}

\section{Participants}

Forty-eight volunteers ( 37 females and 11 males) participated to fulfill a research option in their introductory psychology course at the University of Saskatchewan. The experiment was described as a test of simple arithmetic skills on the participant sign-up sheet. The participants' ages ranged from 17 years to 29 years with a mean age of 19.5 years. In response to the question "What was your first language for arithmetic?" 40 responded "English," 6 responded "French," and one each indicated "Chinese" and "Russian."

\section{Apparatus and Stimuli}

The instructions and stimuli appeared on two high-resolution monitors displayed by an IBM-type personal computer. The experimenter viewed one monitor and the participant viewed the other. Participants wore a lapel microphone connected to a sound-activated relay that controlled a software clock accurate to $\pm 1 \mathrm{msec}$.

Addition problems involved all pairs of single-digit numbers between 2 and 9 (i.e., $2+2$ to $9+9$ ). There are 36 possible pairings of the numbers 2 through 9 when commuted pairs (e.g., $4+5$ and $5+4)$ are counted as one problem. This includes 8 ties (e.g., $2+2$, $3+3$ ) and 28 non-ties (e.g., 3+8, 5+7). The stimuli consisted of Arabic digits $5 \mathrm{~mm}$ high by $3 \mathrm{~mm}$ wide that appeared as white characters against a dark background. The problems were presented horizontally, with the operands separated by the addition sign flanked by blank spaces.

The addition problems were separated into two sets of 18 . Set A included the problems $2+2,2+5,3+4,2+6,3+5,4+4,3+6,2+9$, $4+7,3+9,4+8,5+7,5+8,6+7,6+8,7+7,8+9$, and $9+9$. Set $B$ consisted of $2+3,2+4,3+3,2+7,4+5,2+8,3+7,4+6,5+5$, $3+8,5+6,6+6,4+9,5+9,6+9,7+8,7+9$, and $8+8$. Each number 2 through 9 appeared in four problems in each set. The sets both included 4 tie and 14 non-tie problems, and also had equal representation of even-even, odd-odd, and even-odd combinations.

\section{Design}

The participants received both fast and slow deadline trials, with one problem set (A or B) assigned to each deadline condition. Assignment of the problem sets to fast and slow deadline conditions, and whether the first block tested the slow or the fast deadline, were counterbalanced across participants. The participants were assigned to one of the counterbalancing conditions based on the order in which they entered the experiment (i.e., their participant number). Operand order for non-tie problems (e.g., $5+6$ vs. $6+5$ ) was determined randomly for the first block and then alternated across blocks. The order of problems in each block was independently randomized for each participant.

We did not include a no-report control group in this study (cf. Kirk \& Ashcraft, 2001), because our deadline manipulation was bound to dictate performance regardless of strategy reports. Also, using a strategy selection method essentially identical to that of the present study, Campbell and Timm (2000) compared the performance of two groups $(n \mathrm{~s}=50)$ that did or did not provide strategy reports for 144 multiplication or division problems. There was no evidence that collecting the strategy reports affected performance in any substantial way.

\section{Procedure}

Testing occurred in a quiet room with an experimenter present and required about $25 \mathrm{~min}$. Prior to the arithmetic trials, the participants performed a 20-trial speeded-naming task involving one- or twodigit numbers, which permitted adjustments to the sensitivity of the voice-activated relay. For the arithmetic task, the experimenter explained that there would be six blocks of 18 simple addition trials and that deadline conditions would alternate across blocks. For the fast deadline, the participants were asked to answer before a beep that sounded $750 \mathrm{msec}$ after the problem appeared. For the slow deadline, the participants were asked not to respond until a beep sounded 2,500 msec after problem onset.

The experimenter pressed a key to initiate each block, and a message displayed at the beginning of each block indicated which response deadline would be tested. The participants took a brief break 
between blocks if they wished. Prior to the first block of arithmetic trials, the following instructions appeared on the monitor and were explained by the experimenter:

After each problem please indicate how you solved the problem by choosing from among the following strategies: Transform, Count, Remember, Other. Say TRANSFORM if you used knowledge of a related problem. Say COUNT if you used a strategy based on counting. Say REMEMBER if the answer seemed to come to you without any intermediate steps, inferences, or calculations. Choose OTHER if you used some other strategy or are uncertain

For reference during trials, the participants also received a sheet of strategy descriptions as follows:

Transform: You solve the problem by referring to a related problem in the same or another operation. For example, you might solve 54 / $6=$ ? by remembering that $9 \times 6=54$ so $54 / 9$ must equal 6 .

Count: You solve the problem by counting a certain number of times to get the answer.

Remember: You solve the problem by just remembering or knowing the answer directly from memory without any intervening steps.

Other: You may solve the problem by a strategy unlisted here, or you may be uncertain how you solved the problem.

Prior to each trial, "Beat the beep" or "Wait for the beep" appeared for $1 \mathrm{sec}$ at the middle of the screen. Then a fixation dot appeared and flashed twice over a 1-sec interval at the center of the screen. The problem appeared (synchronized with the monitor's raster scan) on what would have been the third flash with the operation sign $(+)$ at the fixation point. Timing began when the problem appeared and ended when the sound-activated relay was triggered. Triggering the relay caused the problem to disappear immediately. This allowed the experimenter to mark trials that were spoiled because the microphone failed to detect the onset of the response. Immediately after the response, the prompt "Strategy Choice" appeared at the center of the screen with the words Transform, Count, Remember, Other centered horizontally below. The four words were separated by six spaces and always appeared in the same order. The experimenter recorded the strategy reported by pressing one of four buttons on the computer keyboard. Once the strategy was recorded and the experimenter had entered the stated arithmetic answer, the screen cleared and displayed the fixation dot for the next trial. No feedback about speed or accuracy was provided during the experiment.

\section{RESULTS}

\section{Performance}

Our primary interest was in effects of the deadlines on strategy report, but it was important first to determine how successful participants were in meeting the deadline requirements. For the purpose of these analyses, we categorized trials on the basis of the strategy reported into retrieval trials (i.e., Remember selected) and nonretrieval trials (Count, Transform, or Other selected; a complete analysis of the strategy reports follows the performance analyses). Small problems were defined as those with both addends $\leq 5$, else the problem was classified as large (cf. Campbell, 1994; Campbell \& Timm, 2001; Nöel, Fias, \& Brysbaert, 1997). ${ }^{1}$ For each participant, we computed a mean RT for each deadline $\times$ size $\times$ strategy cell after discarding RTs greater than $2.5 S D$ s from each cell. A total of 91 RTs (2\%) were discarded as outliers. Another 382 RTs $(7.4 \%)$ were discarded because of voice key failures.

Approximately half of the participants reported no use of nonretrieval strategies for small problems under either deadline condition. These empty cells precluded a full deadline $\times$ size $\times$ strategy analysis of performance; only 15 of 48 participants provided RT observations in all eight cells. Instead, we performed size $\times$ strategy analyses separately for the fast and slow deadline conditions. For the RT analyses, $n=24$ and $n=21$ for the slow and fast deadline conditions, respectively. For the analysis of errors, $n=29$ for the slow deadline and $n=24$ for the fast deadline. Table 1 presents mean correct RTs (top panel) and mean percentages of errors (bottom panel) as a function of problem size (small or large), deadline (fast or slow), and strategy report (retrieval or nonretrieval). ${ }^{2}$

\section{Slow-Deadline Performance}

RTs. There was no problem size effect observed in the slow-deadline condition, which produced equivalent mean correct RTs for small problems $(3,266 \mathrm{msec})$ and large problems $(3,262 \mathrm{msec})\left[F(1,23)<1, M S_{\mathrm{e}}=30,938.41\right]$. This suggests that the 2,500 -msec deadline usually provided sufficient time to solve both small and large problems, and that answer production RT differences between small and large problems contribute little if anything to the addition problem size effect. In contrast, RTs in the slow-deadline condition were $120 \mathrm{msec}$ slower for nonretrieval $(3,324 \mathrm{msec})$ than for retrieval trials $(3,204 \mathrm{msec})$ $\left[F(1,23)=9.07, M S_{\mathrm{e}}=37,917.91, p=.006\right]$. Nonretrieval strategies generally take longer to complete than retrieval strategies, and this difference emerged despite imposing a 2,500-msec delay before responding. There was no strategy $\times$ size interaction for RTs in the slow deadline condition $\left[F(1,23)<1, M S_{\mathrm{e}}=36,659.46\right]$.

Errors. Error rates were low in the slow-deadline condition, with a weak trend for more errors on nonretrieval trials $(4.7 \%)$ than on retrieval trials $(1.0 \%)[F(1,28)=$ $\left.3.29, M S_{\mathrm{e}}=127.73, p=.081\right]$. As in the RT analysis, there was no evidence of a problem size effect on errors

Table 1

Mean Correct Response Times (in Milliseconds) and Percentages of Errors as a Function of Deadline and Problem Size

\begin{tabular}{|c|c|c|c|c|}
\hline \multirow[b]{3}{*}{ Deadline } & \multicolumn{4}{|c|}{ Problem Size } \\
\hline & \multicolumn{2}{|c|}{ Small } & \multicolumn{2}{|c|}{ Large } \\
\hline & $M$ & $S D$ & $M$ & $S D$ \\
\hline \multicolumn{5}{|c|}{ Response Time for Correct Trials } \\
\hline \multicolumn{5}{|l|}{ Slow $(n=24)$} \\
\hline Retrieval & 3,191 & 158 & 3,217 & 160 \\
\hline Nonretrieval & 3,340 & 304 & 3,307 & 203 \\
\hline \multicolumn{5}{|l|}{ Fast $(n=21)$} \\
\hline Retrieval & 702 & 63 & 839 & 133 \\
\hline \multirow[t]{2}{*}{ Nonretrieval } & 790 & 123 & 1,154 & 437 \\
\hline & \multicolumn{4}{|c|}{ Percentage of Errors } \\
\hline \multicolumn{5}{|l|}{ Slow $(n=29)$} \\
\hline Retrieval & 0.3 & 1.5 & 1.6 & 3.9 \\
\hline NonRetrieval & 6.9 & 22.2 & 2.5 & 3.8 \\
\hline \multicolumn{5}{|l|}{ Fast $(n=24)$} \\
\hline Retrieval & 2.0 & 5.3 & 5.0 & 5.8 \\
\hline Nonretrieval & 9.0 & 23.0 & 21.8 & 19.8 \\
\hline
\end{tabular}

Note $-n=$ number of participants contributing observations to all four size $\times$ strategy cells within each level of the deadline factor. 
Table 2

Mean Percentage Reported Strategy Use as a Function of Deadline (Fast or Slow), Problem Size (Small or Large), and Accuracy

\begin{tabular}{|c|c|c|c|c|}
\hline \multirow[b]{3}{*}{ Strategy } & \multicolumn{4}{|c|}{ Deadline } \\
\hline & \multicolumn{2}{|c|}{ Fast } & \multicolumn{2}{|c|}{ Slow } \\
\hline & $\begin{array}{c}\text { Small } \\
\text { Problem Size }\end{array}$ & $\begin{array}{c}\text { Large } \\
\text { Problem Size }\end{array}$ & $\begin{array}{c}\text { Small } \\
\text { Problem Size }\end{array}$ & $\begin{array}{c}\text { Large } \\
\text { Problem Size } \\
\end{array}$ \\
\hline \multicolumn{5}{|c|}{ Correct Trials } \\
\hline Remember & 84.8 & 71.8 & 84.4 & 63.5 \\
\hline Count & 10.6 & 14.2 & 13.0 & 24.5 \\
\hline Transform & 1.7 & 4.1 & 1.4 & 9.3 \\
\hline Other & 0.2 & 2.7 & 0.6 & 1.2 \\
\hline All & 97.3 & 92.8 & 99.4 & 98.5 \\
\hline \multicolumn{5}{|c|}{ Error Trials } \\
\hline Remember & 2.1 & 3.0 & 0.3 & 0.7 \\
\hline Count & 0.3 & 3.2 & 0.3 & 0.7 \\
\hline Transform & 0.0 & 0.3 & 0.0 & 0.1 \\
\hline Other & 0.4 & 0.7 & 0.0 & 0.1 \\
\hline All & 2.8 & 7.2 & 0.6 & 1.6 \\
\hline
\end{tabular}

Note-Fast-deadline trials with RTs $>900 \mathrm{msec}$ and slow-deadline trials with RTs $<2,500 \mathrm{msec}$ were excluded.

(3.6\% for small problems and $2.1 \%$ for large problems) $\left[F(1,28)<1, M S_{\mathrm{e}}=95.33\right]$. Similarly, there was no strategy $\times$ size interaction for percentage of errors in the slow condition $\left[F(1,28)=1.85, M S_{\mathrm{e}}=131.57, p=.184\right]$.

\section{Fast-Deadline Performance}

RTs. In the fast-deadline condition, the mean RT was substantially faster for retrieval trials $(770 \mathrm{msec})$ than for nonretrieval trials $(972 \mathrm{msec})\left[F(1,20)=14.99, M S_{\mathrm{e}}=\right.$ $57,276.79, p=.001]$. Thus, average time for retrieval trials was close to the $750 \mathrm{msec}$ deadline, whereas responses to nonretrieval trials were given on average about $220 \mathrm{msec}$ after the beep. Unlike the slow deadline condition, there was a large problem size effect, with RTs faster for small problems (746 msec) than for large problems $(997 \mathrm{msec})$ $\left[F(1,20)=33.08, M S_{\mathrm{e}}=40,033.37, p<.001\right]$. There was also a strategy $\times$ size interaction whereby the problem size effect was larger for nonretrieval strategies $(364 \mathrm{msec})$ than for retrieval strategies $(137 \mathrm{msec})$, a difference of $227 \mathrm{msec}\left[F(1,20)=8.46, M S_{\mathrm{e}}=31,930.42, p=.009\right]$.

Errors. The percentages of errors for the fast deadline condition were greater for nonretrieval trials $(15.4 \%)$ than for retrieval trials $(3.5 \%)\left[F(1,23)=18.16, M S_{\mathrm{e}}=\right.$ $188.97, p<.001]$. Small problems $(5.5 \%)$ produced fewer errors than did large problems $(13.4 \%)[F(1,23)=5.4$, $\left.M S_{\mathrm{e}}=276.44, p=.029\right]$. The pattern of the strategy $\times$ size interaction was similar to that observed for RT: The problem size effect tended to be greater for nonretrieval trials $(13 \%)$ than for retrieval trials $(3 \%)$, but the interaction was not significant $\left[F(1,23)=3.20, M S_{\mathrm{e}}=183.46\right.$, $p=.089]$.

Performance in the fast-deadline condition confirmed that the experiment produced typical findings with respect to effects of problem size and strategy (e.g., Campbell \& Timm, 2000; Campbell \& Xue, 2001; LeFevre, Sadesky,
\& Bisanz, 1996). Specifically, large problems were more difficult than small problems, and this difference was exaggerated given nonretrieval strategies relative to when participants reported using direct memory retrieval.

\section{Strategy Reports}

The performance analyses indicated that RTs in the fast-deadline condition often exceeded the 750-msec cutoff by a substantial amount. Potential effects of a response deadline on strategy choice depend, of course, upon participants' adhering to the deadline conditions. Consequently, to test our strategy predictions, we included only fastdeadline trials with RTs $\leq 900 \mathrm{msec}$ and slow-deadline trials with RTs $\geq 2,500 \mathrm{msec}$. We chose $900 \mathrm{msec}$ as the cutoff for the fast deadline because the nominal $750-\mathrm{msec}$ deadline excluded $57 \%$ of all fast-deadline trials and 2 participants were excluded with empty cells. In contrast, only $27 \%$ of the fast-deadline trials were lost with a 900 msec cutoff and all of the 48 participants could be included in the analysis. Of slow-deadline RTs, 93\% and $94 \%$ for small and large problems, respectively, met the slow response requirement.

Table 2 presents the mean percentage reported use of the four strategy categories (i.e., Remember, Count, Transform, Other) as a function of deadline, problem size, and accuracy (i.e., correct vs. error trials). The top half of Table 2 presents the results for correct trials and the bottom half presents the results for error trials. ${ }^{3}$ Our strategy analyses focused on the correct trials, because correct responses accounted for the vast majority of trials, and several error-trial cells were empty.

For correct trials, Remember accounted for $74.0 \%$ of strategy reports, Count for $18.8 \%$, Transform for $5.4 \%$, and Other for the remaining $1.8 \%$. We performed a deadline $\times$ size analysis of the percentage use of Remember. The main effect of problem size reflected the typical finding that direct retrieval is more likely to be reported for small problems $(85 \%)$ than for large problems $(68 \%)$ $\left[F(1,47)=82.42, M S_{\mathrm{e}}=168.21, p<.001\right]$. Importantly, the problem size effect on use of Remember was greater under the slow deadline $(21 \%)$ than under the fast deadline $(13 \%)\left[F(1,47)=7.38, M S_{\mathrm{e}}=100.92, p=.009\right]$. Table 2 shows that reported use of Remember for small problems was the same under the fast $(84.8 \%)$ and slow $(84.4 \%)$ deadlines. In contrast, for large problems, Remember was more likely to be reported under the fast deadline $(72 \%)$ than under the slow deadline (64\%). Thus, as would be expected given that nonretrieval strategies are especially time consuming for larger problems (see Table 1), the fast deadline curtailed use of nonretrieval strategies especially for large problems. This was reflected in both the Count and Transform strategies (see Table 2). The problem size effect on Count as was $11.5 \%$ under the slow deadline but only $3.6 \%$ under the fast deadline. Similarly, for Transform, the problem size effect was larger under the slow deadline $(7.9 \%)$ than under the fast deadline (2.4\%). The results confirm the expected interactive effects of deadline and problem size on strategy use, and 


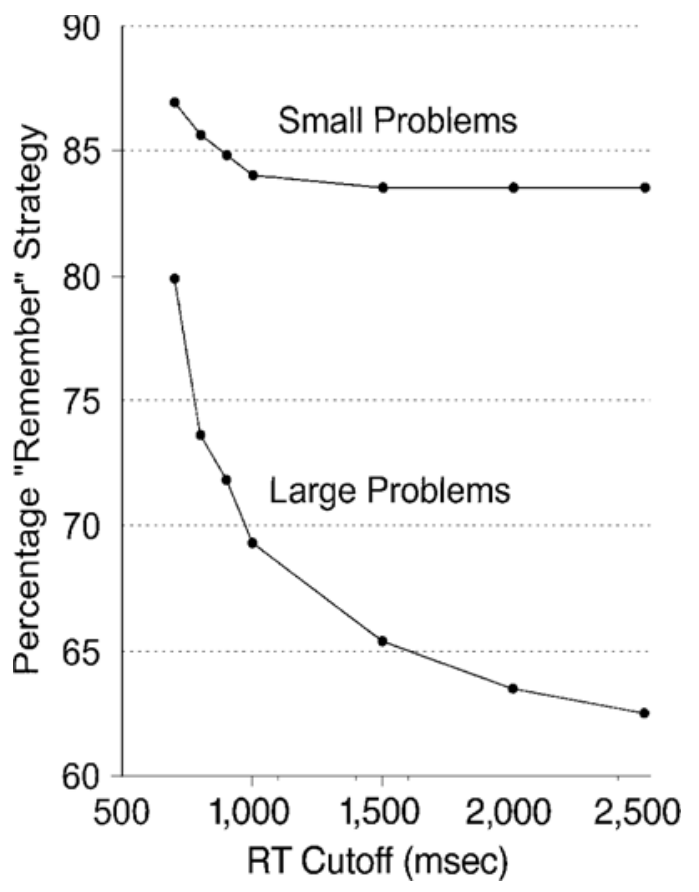

Figure 1. Mean percentage use of Remember on correct trials for small and large problems in the fast-deadline condition, as a function of elapsed time.

they support the conclusion that the strategy selection procedure can distinguish use of direct retrieval from use of nonretrieval procedures for arithmetic.

\section{The Relation Between RT and Use of "Remember"}

The analysis of Table 2 confirmed the predicted deadline $\times$ size interaction on use of Remember, but we can examine the relation between RT and use of Remember more precisely.

Figure 1 presents the mean percentage use of Remember on correct trials for small and large problems in the fastdeadline condition as a function of elapsed time with cutoffs at $700,800,900,1,000,1,500,2,000$, and 2,500 msec. The proportions of fast-deadline trials with RTs below each cutoff were $.34, .57, .73, .83, .94, .97$, and .98 , respectively. As Figure 1 shows, for the small problems, Remember accounted for approximately $85 \%$ of the trials regardless of the RT cutoff, although the Remember rate did increase slightly with cutoffs below $1,000 \mathrm{msec}$. This suggests that procedural strategies for the small problems could usually be completed in $1 \mathrm{sec}$, but that RTs less than $1 \mathrm{sec}$ were increasingly likely to be sampled from the Remember RT distribution. Above $1 \mathrm{sec}$, the Remember rate for small problems was equivalent to that observed under the slow deadline ( $84.4 \%$; see Table 2).

In contrast to that for the small problems, the percentage of Remember for the large problems decreased dramatically as the RT cutoff increased, decreasing from a high of $80 \%$ with a 700 -msec cutoff to a low of $63 \%$ at the 2,500-msec cutoff. Thus, as we would expect given that procedural strategies generally should take longer than direct retrieval, increasing RT was associated with an increased probability of solving a large problem using a procedural strategy. With a 2,500-msec cutoff, the Remember rate for large problems was the same in the fast-deadline condition and in the slow-deadline condition $(63.5 \%$, see Table 2).

\section{DISCUSSION}

This Discussion is focused on two questions. First, What processes underlie the effects of deadline on strategy choice? Second, What are the limits of the strategyselection method?

\section{Effects of Deadline on Strategy Choice}

How might we explain the effects of deadline on strategy choice in our experiment? We assume that simple arithmetic problems activate retrieval processes that provide individuals with information that they use to choose between retrieving an answer and calculating an answer by means of a procedural strategy. The criterion information could be the sense of familiarity produced by recognition processes (Schunn, Reder, Nhouyvanisvong, Richards, \& Stroffolino, 1997) or the associative strengths of answers activated by the problem (Siegler, 1988; Siegler \& Shipley, 1995). People may set a high-activation criterion and use retrieval only when a problem produces high familiarity or strength, or they may set a low-activation criterion that allows retrieval to proceed even when memory produces a weak response to the problem (Siegler \& Campbell, 1990). Here, the speed pressure imposed by the fast deadline would encourage people to set a lower criterion of retrieval strength or familiarity relative to the slow deadline. This is because procedural strategies were difficult to complete within the fast deadline, and retrieval generally is faster (about $300 \mathrm{msec}$ faster for the large additions; see Table 1). Setting a lower retrieval criterion would increase the probability of providing a response within (or close to) the deadline by increasing use of retrieval.

In general, small-number problems are expected to generate stronger activation or familiarity than are largenumber problems (Campbell \& Xue, 2001; Siegler \& Shrager, 1984). Consequently, we expect more use of retrieval for small problems than for large problems, as was observed. Greater memory activation for small than for large problems also implies that lowering the retrieval criterion would affect strategy choice less for small problems than for large problems. Indeed, the fast deadline had little effect on retrieval usage for small problems, which were solved predominantly by retrieval under both deadlines $(84 \%)$. This suggests that the criterion was adjusted specifically to accommodate the larger problems, which were especially difficult to solve by procedural strategies under the fast deadline. Indeed, attempts to complete a procedural strategy for large problems under the fast dead- 
line often failed (see Table 1) presumably because insufficient time was available for one to accurately complete a multistep procedure. Adopting a lower criterion for retrieval increased retrieval for large problems by about $17 \%$ on the average in the fast-deadline condition relative to the slow-deadline condition (see Table 2, including both correct and error trials; see also Figure 1). This would be an adaptive response to the fast deadline (cf. Siegler \& Lemaire, 1997), because, for large problems, retrieval was both substantially faster and more accurate than were nonretrieval strategies (Table 1).

\section{Utility of the Strategy Selection Method}

The effects of our deadline manipulation on reported use of retrieval suggest that the strategy selection procedure can potentially discriminate different strategies for simple arithmetic. Nonetheless, the precision and reliability of the strategy data remain uncertain. Although our instructions did not emphasize one strategy over another, the mere fact of having provided a specific set of strategy categories may introduce demand characteristics that bias strategy reports (Kirk \& Ashcraft, 2001). If the strategy selection method were heavily biased by demand characteristics, however, we would expect it to present a rather limited range of strategy profiles. But this is not the case. Using the strategy selection method (i.e., select from Transform, Count, Remember, Other), Campbell and Xue (2001) found that Asian Chinese university students reported retrieval rates of $100 \%$ for multiplication, $92 \%$ for addition, $79 \%$ for subtraction, and $71 \%$ for division. NonAsian Canadian students reported retrieval rates of $97 \%$ for multiplication, $76 \%$ for addition, $58 \%$ for subtraction, and $57 \%$ for division. The problem size effect on retrieval usage ranged from $0 \%$ up to $31 \%$. Thus, despite the possibility of demand characteristics, the strategy selection method is apparently capable of discriminating a broad range of strategy profiles with respect to usage of retrieval versus procedural strategies.

The strategy selection method produces estimates of retrieval usage that are consistent with the results of full-report methods (see Campbell \& Xue, 2001, pp. 310-311). Furthermore, percentage use of retrieval as estimated by the strategy selection method accounts coherently for RT differences across arithmetic operations, problem characteristics, and cultural groups (Campbell \& Xue, 2001), as well as across a variety of experimental manipulations, including the RT deadlines of the present experiment, retrieval interference (Campbell \& Timm, 2000), and manipulation of surface notation (Campbell \& Fugelsang, 2001). On the basis of these observations, we have concluded that the strategy selection method is a useful, if imperfect, tool for analyzing strategic aspects of adults' simple arithmetic skills.

It is important to emphasize, however, that there has been considerable variability in estimates of adults' strategies for simple arithmetic (see Campbell \& Xue, 2001,pp. 310311 , for a recent review). The variability across studies in reported usage of retrieval probably arises because strategy usage and reports are influenced by many factors, including population or sampling differences, speed-accuracy criteria, experimental design, and strategy instructions (Ericsson \& Simon, 1993; White, 1994). Indeed, the potential for bias in strategy instructions cannot be overemphasized (cf. Kirk \& Ashcraft, 2001). For example, in the present experiment, selection of Count accounted for $19 \%$ of trials; of Transform, 5\%. In contrast, for their nonAsian Canadian group (the group most analogous to our current sample) Campbell and Xue found the reverse: a lower rate of Count (5\%) than Transform (19\%) for simple addition (see also Geary \& Wiley, 1991; LeFevre, Sadesky, \& Bisanz, 1996). The inconsistent results for transformation and counting probably reflect differences across experiments in instructions to participants. Specifically, Campbell and Xue's strategy instructions emphasized that Count applied strictly to solving the problem by counting by ones, whereas the instructions used in the present study did not stipulate this. Transformation strategies may include a substantial counting component (e.g., $6+8$ is $6+6=12+[8-6=2]+1=13+1=14)$; and our participants would have been free to select Count for any trial that included a counting element. Thus, we think that the Count category was used both for "pure" counting strategies and for transformation strategies that included a counting element. This would explain the higher rate of Count relative to Transform in our study (cf. Campbell \& Timm, 2000) than in Campbell and Xue. Such inconsistencies highlight the importance of precise experimental instructions to the validity and interpretation of strategy reports for arithmetic.

\section{REFERENCES}

Ashcraft, M. H. (1992). Cognitive arithmetic: A review of data and theory. Cognition, 44, 75-106.

AsHCRAFT, M. H. (1995). Cognitive psychology and simple arithmetic: A review and summary of new directions. Mathematical Cognition, 1, 3-34.

Ashcraft, M. H., \& Christy, K. S. (1995). The frequency of arithmetic facts in elementary texts: Addition and multiplication in Grades 1-6. Journal for Research in Mathematics Education, 5, 396-421.

CAmpbell, J. I. D. (1994). Architectures for numerical cognition. Cognition, 53, 1-44.

Campbell, J. I. D., \& Fugelsang, J. (2001). Strategy choice for arithmetic verification: Effects of numerical surface form. Cognition, $\mathbf{8 0}$, B21-B30.

Campbell, J. I. D., \& Timm, J. C. (2000). Adults' strategy choices for simple addition: Effects of retrieval interference. Psychonomic Bulletin \& Review, 7, 692-699.

Campbell, J. I. D., \& Xue, Q. (2001). Cognitive arithmetic across cultures. Journal of Experimental Psychology: General, 130, 299-315.

Ericsson, K. A., \& Simon, H. A. (1993). Protocol analysis: Verbal reports as data (rev. ed.). Cambridge, MA: MIT Press.

Geary, D. C. (1996). The problem-size effect in mental addition: Developmental and cross-national trends. Mathematical Cognition, 2, 63-93.

Geary, D. C., Frensch, P. A., \& Wiley, J. D. (1993). Simple and complex mental subtraction: Strategy choice and speed-of-processing differences in younger and older adults. Psychology \& Aging, 8, 242-256. Geary, D. C., \& Wiley, J. G. (1991). Cognitive addition: Strategy 
choice and speed-of-processing differences in young and elderly adults. Psychology \& Aging, 6, 474-483.

Hamann, M. S., \& Ashcraft, M. H. (1986). Textbook presentations of the basic addition facts. Cognition \& Instruction, 3, 173-192.

HECHT, S. A. (1999). Individual solution processes while solving addition and multiplication math facts in adults. Memory \& Cognition, 27, 1097-1107.

Kirk, E. P., \& AshCraft, M. H. (2001). Telling stories: The perils and promise of using verbal reports to study math strategies. Journal of Experimental Psychology: Learning, Memory, \& Cognition, 27, 157175.

LeFevre, J.-A., Bisanz, J., Daley, K. E., Buffone, L., Greenham, S. L., \& SADESKY, G. S. (1996). Multiple routes to solution of single-digit multiplication problems. Journal of Experimental Psychology: General, 125, 284-306.

LeFevre, J.-A., \& LiU, J. (1997). The role of experience in numerical skill: Multiplication performance in adults from China and Canada. Mathematical Cognition, 3, 31-62.

LeFevre, J.-A., \& Morris, J. (1999). More on the relation between division and multiplication in simple arithmetic: Evidence for mediation of division solutions via multiplication. Memory \& Cognition, 27, 803-812.

LeFevre, J.-A., Sadesky, G. S., \& Bisanz, J. (1996). Selection of procedures in mental addition: Reassessing the problem size effect in adults. Journal of Experimental Psychology: Learning, Memory, \& Cognition, 22, 216-230.

NöEL, M.-P., Fias, W., \& Brysbaert, M. (1997). About the influence of the presentation format on arithmetical-fact retrieval processes. $\mathrm{Cog}$ nition, 63, 335-374.

Robinson, K. M. (2001). The validity of verbal reports in children's subtraction. Journal of Educational Psychology, 93, 211-222.

Schunn, C. D., Reder, L. M., Nhouyvanisvong, A, Richards, D. R, \& Stroffolino,P. J. (1997). To calculate or not to calculate: A source activation confusion model of problem familiarity's role in strategy selection. Journal of Experimental Psychology: Learning, Memory, \& Cognition, 23, 3-29.

SIEGLER, R. S. (1988). Strategy choice procedures and the development of multiplication skill. Journal of Experimental Psychology: General, 117, 258-275.

Siegler, R. S., \& CAMPbell, J. I. D. (1990). Diagnosing individual dif- ferences in strategy choice procedures. In N. Fredriksen (Ed.), Diagnostic monitoring of skill and knowledge acquisition (pp. 113-139). Hillsdale, NJ: Erlbaum.

Siegler, R. S., \& Lemaire, P. (1997). Older and younger adults' strategy choices in multiplication: Testing predictions of ASCM using the choice/no-choice method. Journal of Experimental Psychology: General, 126, 71-92.

Siegler, R. S., \& Shipley, C. (1995). Variation, selection, and cognitive change. In G. Halford \& T. Simon (Eds.), Developing cognitive competence: New approaches to process modelling (pp. 31-76). Hillsdale, NJ: Erlbaum.

Siegler, R. S., \& Shrager, J. (1984). A model of strategy choice. In C. Sophian (Ed.), Origins of cognitive skills (pp. 229-293). Hillsdale, NJ: Erlbaum.

WhITE, T. D. (1994). The proper protocol: Validity and completeness of verbal reports. Psychological Science, 5, 249-252.

\section{NOTES}

1. Both Sets A and B have equal numbers of small and large problems when "small" is defined as problems with both addends $\leq 5$. Equivalent results were obtained defining small as problems with sums $\leq 10$ (cf. LeFevre, Sadesky, \& Bisanz, 1996) or products $\leq 25$ (Campbell \& Xue, 2001).

2. Although the means in Table 1 are based, in some cases, on fewer than half of the participants, they nonetheless were very close to the values obtained when all available participants were included in the mean for each individual cell. Across the eight cells in Table 1, the mean absolute RT deviation between Table 1 means and all available participant means was $17 \mathrm{msec}(S D=8.7)$. For percentage of errors, the mean absolute deviation was $0.8 \%(S D=1.4)$.

3. The error percentages in Table 1 differ from those in Table 2. Table 2 presents the mean percentages of all trials in each size $\times$ deadline condition that involved Remember errors, Count errors, Transform errors, or Other errors. In contrast, Table 1 presents the percentages of errors conditionalized on strategy (i.e., retrieval vs. nonretrieval trials).

(Manuscript received February 6, 2002; revision accepted for publication April 26, 2002.) 